\title{
Improvement of the mechanical response of an electrical component
}

\author{
F.J.V. Antunes*, J.D.M. Costa, J.A.M. Ferreira \\ Department of Mechanical Engineering, University of Coimbra, Pólo II, Pinhal de Marrocos, \\ 3030 Coimbra, Portugal
}

Received 13 March 2003; accepted 26 March 2003

\begin{abstract}
The electrical component under analysis does not fulfil the mechanical performance required by the standard (a minimum force of $400 \mathrm{gf}$ during the extraction of a $3.8 \mathrm{~mm}$ diameter pin after the introduction of a $5.1 \mathrm{~mm}$ pin). A numerical study using the finite element method was developed to understand the limited response of the component and to study the effect of changing material and geometry. Experimental tests were performed to obtain the elasticplastic behaviour of the material and friction coefficient. The poor performance was explained by the exaggerated plastic deformation. The best solution was found to be the use of a new material (extra-hard brass) since the geometrical changes allowed have a limited effect.
\end{abstract}

(C) 2003 Elsevier Ltd. All rights reserved.

Keywords: Plastic deformation; Geometry optimisation; Finite element analysis; Electrical-component failures; Friction

\section{Introduction}

Fig. 1 is a photograph of the electrical component under analysis, produced by EFAPEL —Empresa Fabril de Produtos Eléctricos. It is an electrical socket, constituted by a plastic support and two brass pieces (there is only one in the assembly of Fig. 1). This component does not fulfil the standard DIN VDE 0620: a minimum friction force of $400 \mathrm{gf}$ is required during the extraction of a $3.8 \mathrm{~mm}$ diameter pin after the introduction of a $5.1 \mathrm{~mm}$ pin.

The objectives of this paper are to understand the reasons for the limited response of the component, and to propose and study solutions that ensure a minimum friction force of $400 \mathrm{gf}$. An alternative material and different geometries were studied. The geometry of plastic support could not be changed, which imposed limitations to geometrical changes.

A numerical procedure was developed using the finite element method to predict the friction force. The finite element method program MSC. MARC MENTAT 2000 was used and elastic-plastic behaviour was assumed for the material. Experimental tests were performed to obtain the elastic-plastic behaviour of the

\footnotetext{
* Corresponding author. Tel.: +351-239-790700; fax: + 351-239-790701.

E-mail address: fernando.ventura@dem.uc.pt (F.J.V. Antunes).
} 


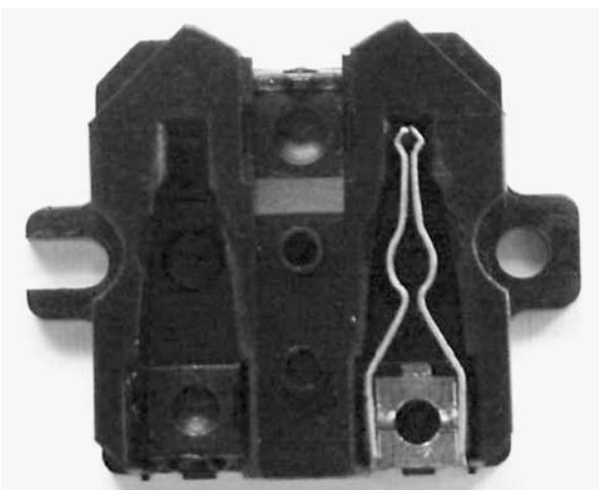

Fig. 1. Plastic support and brass socket.

material and the friction coefficient for the pair steel/brass. The geometry and material being used at EFAPEL were analysed numerically and the friction force obtained was compared with experimental values for validation. After that the geometry and material were changed in order to study their influence on friction force and to obtain a solution to the problem.

\section{Experimental results}

\subsection{Stress-strain curves}

The numerical analysis of elastic-plastic behaviour requires the knowledge of the stress-strain curve. Therefore tension specimens were cut from the band used to produce the brass component [1]. Fig. 2 shows these two directions and the shape of the specimens. Two materials (hard brass and extra-hard brass) and two directions (longitudinal and transversal to the sheet) were studied. The specimens were tested to rupture using an Instron electromechanical tension testing machine; load was measured using a load cell while deformation was measured using an axial extensometer.

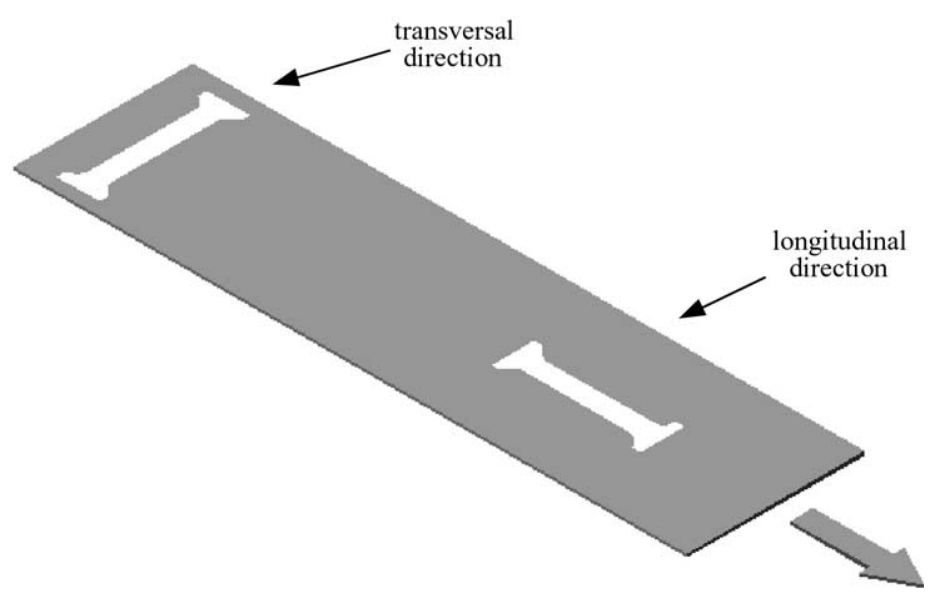

Fig. 2. Longitudinal and transversal directions relative to brass band. 
The results are presented in Table 1 and Fig. 3. It can be seen that extra-hard brass is more resistant than hard brass with higher values of $E$ (Young's modulus) and $\sigma_{\mathrm{y}}$ (yield stress). Both materials are more resistant along the transversal direction than along the longitudinal direction but the difference is more important for extra-hard brass. Both materials have an almost elastic-perfectly plastic behaviour. The hardness was also measured and values of 170 and 212 HV2 were obtained for hard and extra-hard brass, respectively.

\subsection{Steel/brass friction coefficient}

The friction coefficient between the steel pin and the brass component was determined experimentally measuring normal and tangential forces at low relative speed using a cylinder/plane geometry. The normal and friction forces were continuously measured using two load cells. A stable value of 0.21 was obtained for the friction coefficient.

\subsection{Experimental determination of friction force}

The friction force between the brass component and the pin was determined experimentally for the component produced by EFAPEL. Average values ranging from 300 to $500 \mathrm{gf}$ were obtained. This great

Table 1

Material properties

\begin{tabular}{|c|c|c|c|c|c|c|}
\hline & \multicolumn{3}{|c|}{ Hard brass } & \multicolumn{3}{|c|}{ Extra-hard brass } \\
\hline & $E(\mathrm{MPa})$ & $\sigma_{\mathrm{y}}(\mathrm{MPa})$ & $v$ & $E(\mathrm{MPa})$ & $\sigma_{y}(\mathrm{MPa})$ & $v$ \\
\hline Longitudinal & 128150 & 290 & 0.32 & 150300 & 390 & 0.31 \\
\hline Transversal & 128500 & 360 & 0.32 & 110800 & 420 & 0.31 \\
\hline
\end{tabular}

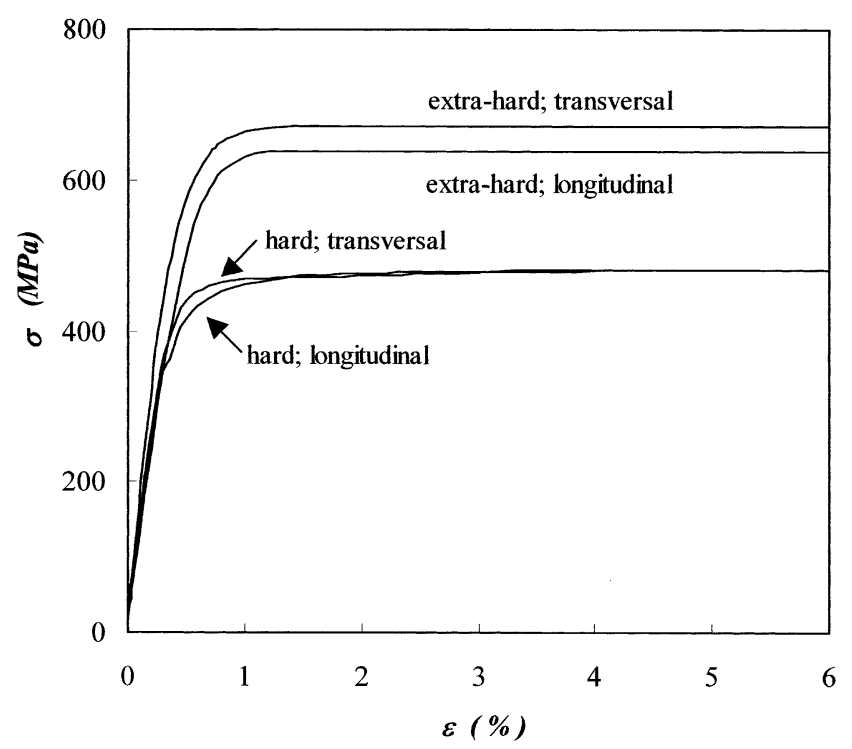

Fig. 3. Stress-strain curves for hard and extra-hard brass along transversal and longitudinal directions. 
variation was explained by the geometry of the support, which was found to vary significantly. Lower friction forces were obtained when the brass piece was smaller than the space in the support, while zero gaps produce friction forces higher than the minimum required. The geometrical variations of the brass components seem to be less significant.

\section{Numerical procedure}

\subsection{Physical model}

Fig. 4 presents the geometry of brass piece, which is being produced by EFAPEL. The component is symmetrical in terms of geometry, material and boundary conditions, therefore only half of it was analysed considering adequate boundary conditions. The plastic support was simulated considering rigid walls where the component contacts. The application of the pins was simulated considering cylindrical pieces, as can be seen in Fig. 4. A pin with $5.1 \mathrm{~mm}$ diameter was first moved onto the specimen and removed subsequently. Then a $3.8 \mathrm{~mm}$ diameter pin was applied and the normal force measured. The friction force was obtained multiplying by the friction coefficient by 2 , since only half of the geometry is being simulated (friction force was calculated assuming Coulomb's model).

The material was assumed to be continuous, homogeneous and isotropic. Von Mises yield criterion was assumed along with a kinematic hardening. The back-stress was defined by Ziegler's hardening rule [2,3]. The elastic-plastic properties presented in Fig. 3 and Table 1 for the transversal direction were considered. This direction was chosen considering the orientation of the brass component on the band of raw material. For the pins a linear elastic behaviour was considered, with $E=210 \mathrm{GPa}$ (Young's modulus) and $v=0.3$ (Poisson's ratio), typical values for steel.

The main limitations of this model are:

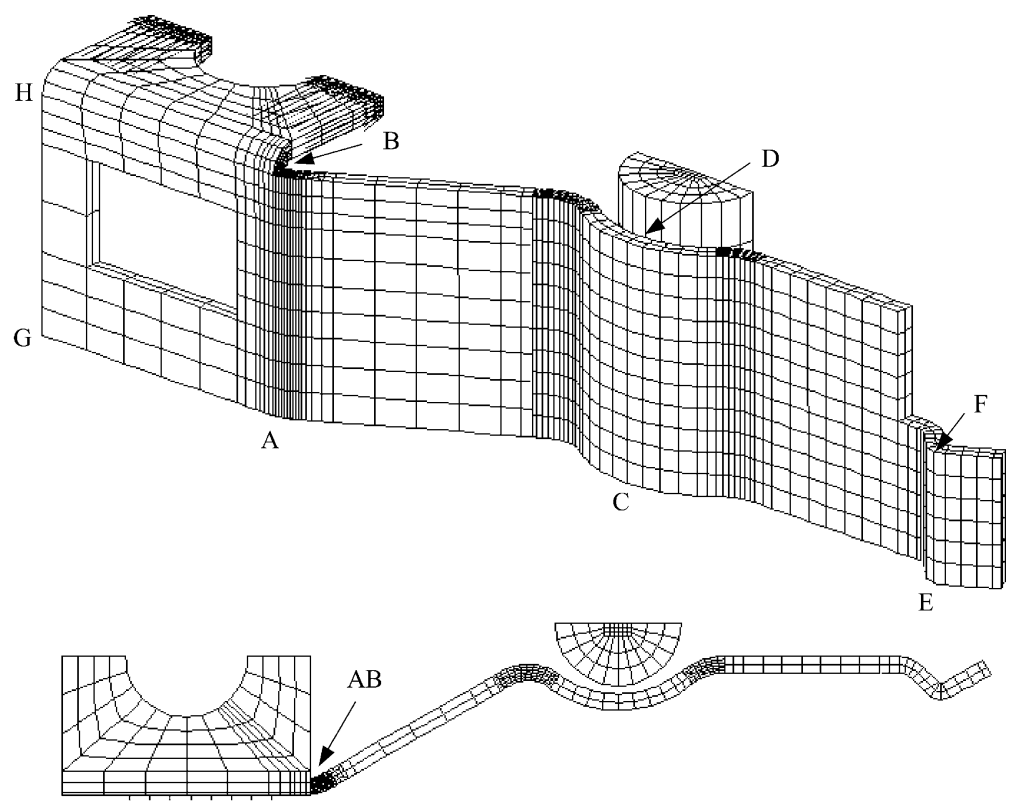

Fig. 4. Geometry of electrical component and $3.8 \mathrm{~mm}$ pin. 
- the isotropic behaviour assumed. In fact, raw material is produced by lamination therefore an anisotropic behaviour can be expected;

- previous hardening resulting from stamping is not considered. This previous plastic deformation reduces posterior plastic deformation.

\subsection{Analysis by the finite element method}

The numerical study was developed using the finite element method program MSC.MARC MENTAT 2000 [4]. Large displacements and large deformations were considered. The non-linear equations were solved using the Newton-Raphson iterative method, which was stopped when maximum residual force/ maximum reaction force $<0.05 \%$.

The finite elements used were the 20-node isoparametric ones with reduced integration. Fig. 4 shows the finite element mesh considered, which had 4028 elements and 20236 nodes. Smaller elements were considered in stress concentration regions.

\section{Numerical results}

\subsection{Initial geometry}

Fig. 5 shows the defomation of the brass component by the $3.8 \mathrm{~mm}$ diameter pin after the application of a $5.1 \mathrm{~mm}$ diameter pin. The numerical simulation of the component which is being produced by EFAPEL produced a friction force of $383 \mathrm{gf}$, which is lower than the minimum force required. This value was obtained considering $d_{1}=0.1 \mathrm{~mm}$ (gap existing between the extremity of the component and the plastic support). This prediction is lower than experimental results obtained for reduced gaps between brass component and support, which can be explained by the previous hardening produced by stamping (not considered in the numerical analysis).

The introduction of a pin imposes a fixed displacement at section $C D$ (Fig. 4), which deforms all the component, as can be seen in Fig. 5. The component contacts with the plastic support at section $E F$ and at the vertical wall $A B H G$, therefore it can be seen as a simply supported beam at $E F$ (see Fig. 4). The horizontal material restrains the rotation at section $\mathrm{AB}$, particularly near point $B$. The rigidity of the component produces a normal force on the pin and consequently the occurrence of a friction force when the pin is being removed.

The limited response of the component can be explained by exaggerated plastic deformation produced by the introduction of the $5.1 \mathrm{~mm}$ diameter pin. This plastic deformation is particularly important at section $A B$ (see Fig. 4). The zone close to point $B$ is more critical as the constraint imposed by the horizontal material forces the occurrence of plastic deformation. This explains why after the introduction of the 5.1 $\mathrm{mm}$ pin the $3.8 \mathrm{~mm}$ pin contacts near point $C$ and does not contact near point $D$.

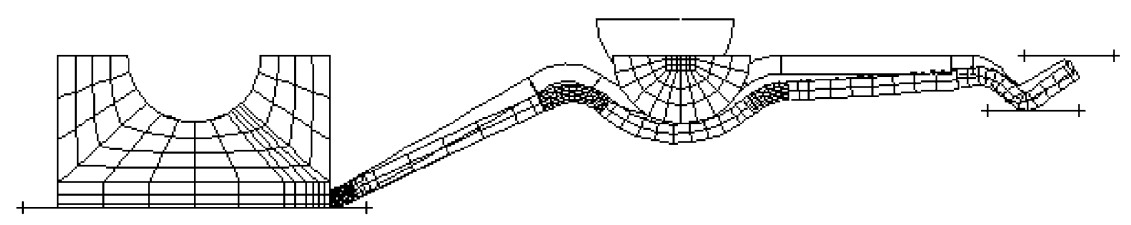

Fig. 5. Initial and deformed shapes. 


\subsection{Effect of material}

The replacement of hard brass by extra-hard brass produces an important increase of the friction force $(+250 \%)$, as can be seen in Fig. 6. This result could be expected considering material properties (Fig. 3 and Table 1). In fact, extra-hard brass has higher Young's modulus and yield stress, therefore a lower level of plastic deformation is observed and the material is more stiff. In fact, the selection of the material for this application is an interesting problem. The material should have a high yield stress to reduce plastic deformation, and an intermediate value of Young's modulus $(E)$. For a low value of $E$ the component would have a reduced stiffness therefore the friction force would be too small even without plastic deformation. On the other hand, an exaggerated value of $E$ would give a high friction force but would require an enormous force to introduce the pin. Brass has a reasonable value of $E$ and also good electrical properties.

So, the use of extra-hard brass would solve the problem. However, this material is more expensive and more difficult to work with, therefore geometrical changes were studied numerically in order to find a solution with hard brass. The main objective of these changes is to reduce the plastic deformation at zone $B$ (Fig. 4) without introducing it at other positions and without reducing significantly the rigidity of the component.

\subsection{Effect of geometrical changes}

Fig. 7 presents the geometrical parameters studied. $d_{1}$ is the gap existing between the extremity of the component and the plastic support, $d_{2}$ is the distance between section $A B$ and the center of the pin, and $d_{3}$ measures the distance between the extremity of the component and the zone where the pin is introduced.

A limited influence of $d_{1}$ on friction force can be seen in Fig. 6 for hard and extra-hard brass compared with the influence of material. Maximum friction forces were obtained for $2 * d_{1}=0.4 \mathrm{~mm}$. Fig. 8 presents the influence of $d_{3}$ on friction force for $d_{1}=0.1 . \Delta d_{3}$ is the increment of distance $d_{3}$ relatively to the size being produced at EFAPEL. The increase of this parameter increases significantly the friction force, particularly for hard brass. Fig. 8 also compares results for an increment of $d_{2}\left(\Delta d_{2}\right)$ of $1.2 \mathrm{~mm}$ with initial geometry $\left(\Delta d_{2}=0\right)$. The increase of $d_{2}$ increases the friction force significantly. The increase of $d_{1}, d_{2}$ and $d_{3}$

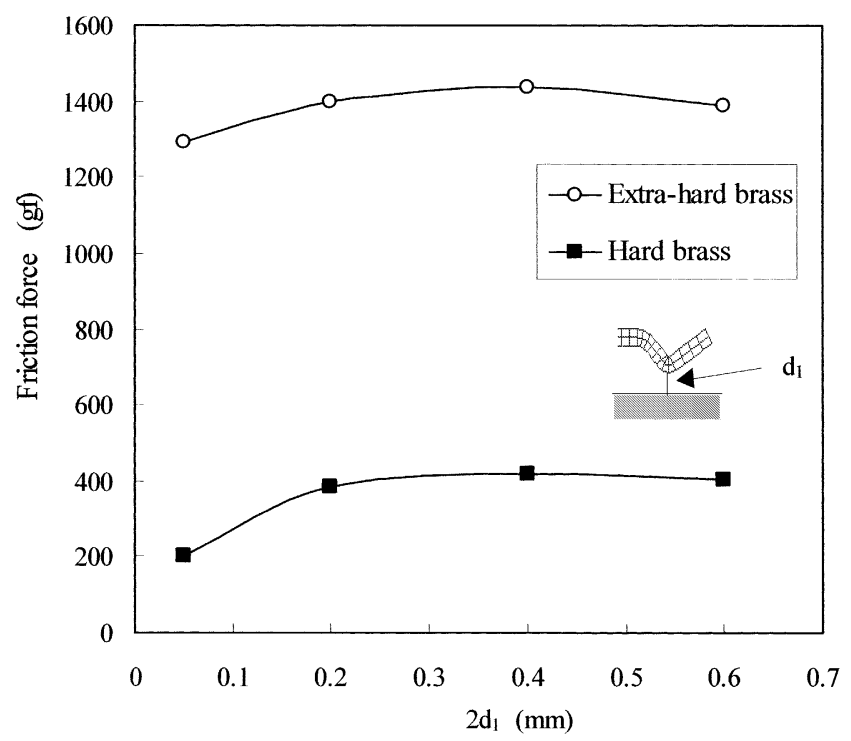

Fig. 6. Geometrical parameters. 
reduces plastic deformation produced by the $5.1 \mathrm{~mm}$ diameter pin at section $\mathrm{AB}$, because the deflection angle imposed is reduced. Therefore the elastic recovering is more significant and higher friction forces are obtained. On the other hand the increase of these parameters reduce the rigidity of the component to the introduction of steel pins, reducing normal and friction forces. The increase of $d_{2}$ and $d_{3}$ have a positive influence which indicates that the reduction of plastic deformation is dominant.

Another modification studied was the reduction of the distance between the pin and the zone where the brass piece first contacts the plastic support (Fig. 9). The objective was to reduce the constraint imposed by the horizontal material on section $A B$. The distance $d_{2}$ was maintained constant while a distance $d_{4} \neq 0$ was

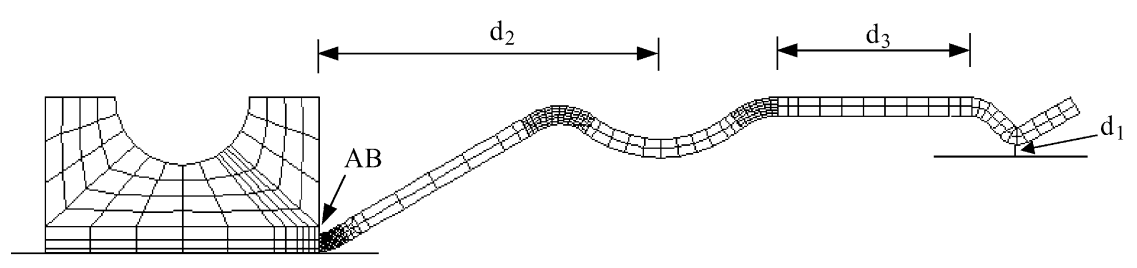

Fig. 7. Geometrical parameters.

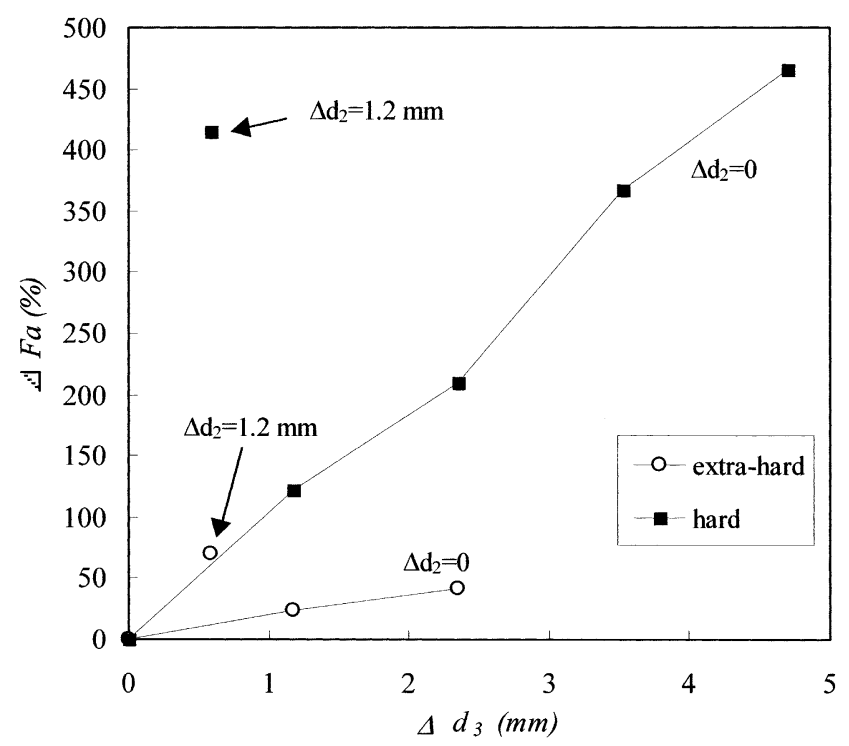

Fig. 8. Influence of $d_{2}$ and $d_{3}$ on friction force.

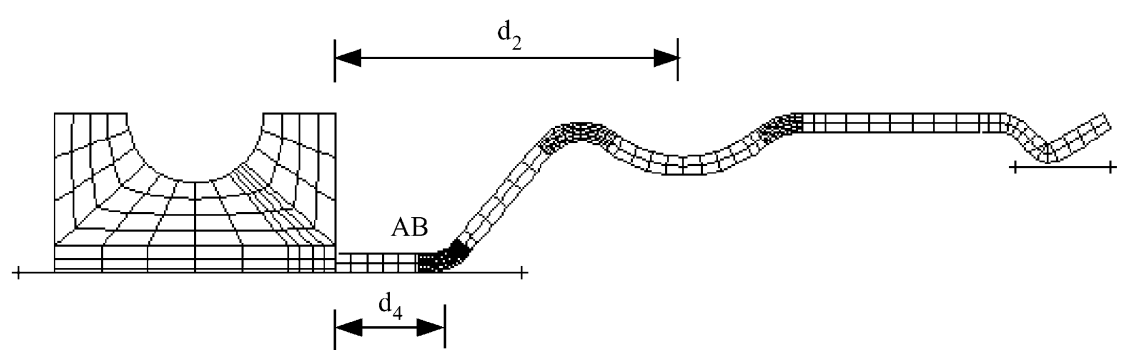

Fig. 9. Consideration of a distance $d_{4} 0$. 
considered. The plastic deformation at $A B$ increased significantly, and the $3.8 \mathrm{~mm}$ pin did not contact the brass component. Therefore this change is negative and must be avoided.

So, the numerical results indicate that the increase of distances $d_{2}$ and $d_{3}$ (Fig. 7) increase the friction force, the influence of $d_{2}$ being more important. Smooth concordances are also expected to improve the performance of the component because stress concentration factors are reduced.

\subsection{A new geometry}

A new geometry was defined considering these recommendations and also the limitations imposed by the plastic support. Fig. 10 presents this new geometry. The concordances were smoothed and material was removed near point $B$ to reduce the constraint imposed to the rotation of vertical wall by the horizontal material. Unfortunately distance $d_{2}$ and $d_{3}$ could not be changed because the plastic support was maintained.

Fig. 11 compares the results obtained with this geometry and the initial geometry. This new geometry gave better results for extra-hard brass but worse results for hard brass. These poor results are explained

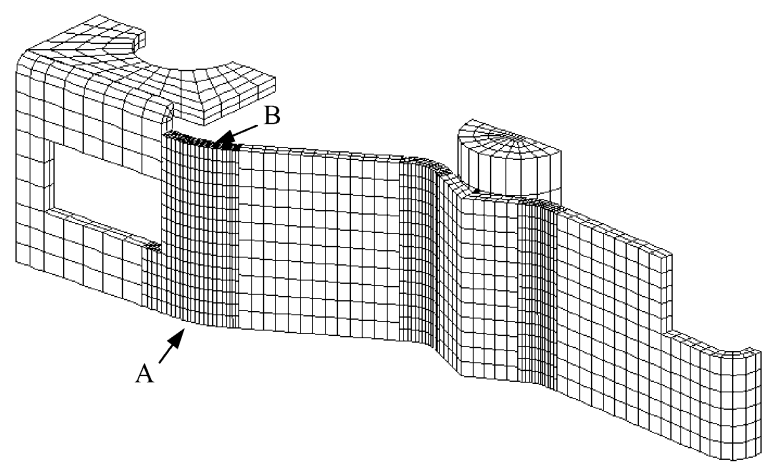

Fig. 10. New geometry.

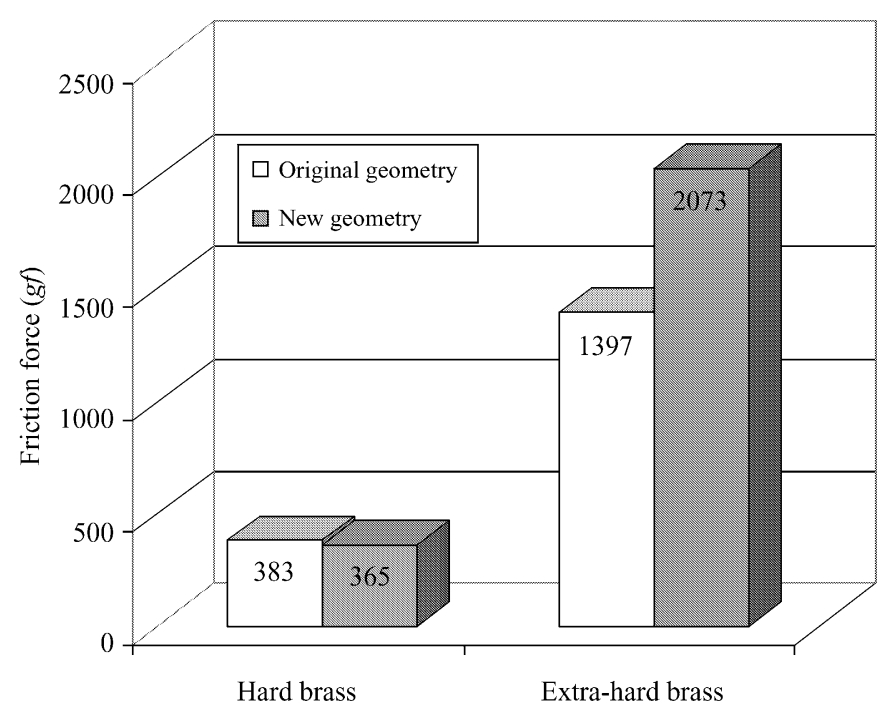

Fig. 11. Comparison between the original and new geometries. 
mainly by the proximity of the window at vertical wall $A B H G$ relatively to section $A B$. It would be interesting to reduce this window, however this would need the modification of the support.

\section{Conclusions}

The numerical results obtained indicate that the poor performance of the brass component is due to exaggerated plastic deformation produced by the introduction of the $5.1 \mathrm{~mm}$ diameter pin. This plastic deformation has a great extent at section $A B$ (Fig. 4) and particularly near point $B$ due to the constraint imposed by the horizontal material.

To obtain the friction force needed (400 gf) it is recommended to use a new material (extra-hard brass). The hard brass along with adequate geometrical changes also gives the minimum friction force. However, the plastic support cannot be changed and this imposes important limitations to the geometrical changes admitted. A new geometry was proposed but the results indicate that it is adequate only for extra-hard brass.

\section{Acknowledgements}

The authors acknowledge EFAPEL (Empresa Fabril de Produtos Eléctricos), Lousã-Portugal for financial support and Prof Amílcar Ramalho for the calculation of friction force.

\section{References}

[1] NP EN 10 002-1. Norma Portuguesa. Metallic materials: tension test. Part 1: testing method at room temperature. 1990.

[2] Khan AS, Huang S. Continuum theory of plasticity. John Wiley \& Sons; 1995.

[3] Ziegler H. A modification of Prager's hardening rule. Quart Appl Math 1959;17:55.

[4] MARC User Information. Palo Alto: Marc Analysis Research Corp.; 2000. 